\title{
Estudo do comportamento reológico das argilas bentoníticas de Cubati, Paraíba, Brasil
}

\author{
(Rheological behavior study of bentonite \\ clays from Cubati, Paraíba, Brazil)
}

\author{
R. R. Menezes, L. F. A. Campos, H. S. Ferreira, L. N. Marques, G. A. Neves, H. C. Ferreira \\ Unidade Acadêmica de Engenharia de Materiais, Universidade Federal de Campina Grande \\ Av. Aprígio Veloso 882, Bodocongó, Campina Grande, PB 58109-970 \\ romualdomenezes@dema.ufcg.edu.br,gelmires@dema.ufcg.edu.br
}

\begin{abstract}
Resumo
O estado da Paraíba possui jazidas de bentonitas utilizadas comercialmente para uma vasta gama de setores tecnológicos. No entanto, esses jazimentos estão se exaurindo, após dezenas de anos de exploração. Assim, este trabalho tem por objetivo dar prosseguimento à caracterização de argilas bentoníticas de Cubati, $\mathrm{PB}$, estudando o comportamento reológico das dispersões argila-água de modo avaliar seu desempenho para fluidos de perfuração de poços de petróleo base água. As amostras estudadas foram secas a $60{ }^{\circ} \mathrm{C}$ e determinou-se sua capacidade de troca de cátions. As argilas foram transformadas em sódica por tratamento com $\mathrm{Na}_{2} \mathrm{CO}_{3}$ e em seguida realizou-se o estudo do comportamento reológico das dispersões. As viscosidades, aparente e plástica, o volume de filtrado, os parâmetros reológicos do fluido e a sua força gel foram determinados. Os resultados evidenciaram que cada amostra apresenta um teor ótimo de carbonato de sódio e que as dispersões argila-água possuem um comportamento pseudo-plástico e tixotrópico. Duas amostras apresentam potencial para utilização como agentes viscosificantes para fluidos de perfuração.
\end{abstract}

Palavras-chave: bentonita, reologia, caracterização, fluidos de perfuração.

\begin{abstract}
The bentonites of the state of Paraíba, Brazil, are commercially used in numerous technological sectors, particularly in oil drilling muds. However, these bentonite deposits are becoming exhausted due to several years of exploitation. Thus, the aim of this work was continue the characterization of the bentonites from Cubati, Paraiba, studying the rheological behavior of water-clay suspensions, evaluating their viability to be used in oil drilling muds. The samples were dried at $60^{\circ} \mathrm{C}$ and, their cation exchange capacity was determined. The natural bentonite clays were transformed in sodium bentonite by addition of concentrated $\mathrm{Na}_{2} \mathrm{CO}_{3}$ solution, then the suspensions rheological study was performed. The apparent and plastic viscosities, water loss, rheological parameters and the gel force were determined. Results showed that each sample has an optimum sodium carbonate content and that the waterclay suspensions presented a pseudo-plastic and tixotropic behavior. The rheological properties show that two samples present technological potential to be used in drilling muds.
\end{abstract}

Keywords: bentonite, reology, characterization, drilling muds.

\section{INTRODUÇÃO}

As argilas esmectitas são materiais constituídos por um, ou mais, argilominerais esmectíticos e por alguns minerais acessórios (principalmente quartzo, cristobalita, micas e feldspatos) [1]. Os argilominerais esmectíticos caracterizamse por apresentarem dentro de sua estrutura cristalográfica elevado grau de substituição isomórfica, o que origina um excesso de carga negativa que são compensadas por cátions interlamelares [2]. De acordo com os cátions de compensação presentes na argila, tem-se que as argilas esmectíticas podem ser monocatiônicas ou policatiônicas, com predominância de um cátion ou não $[1,3]$. Tecnologicamente as argilas esmectíticas são geralmente denominadas bentonitas [1,4].
As argilas bentoníticas são largamente utilizadas em muitos setores industriais, sendo incluídas na classe dos minerais de maior interesse industrial $[5,6]$. No Brasil, as argilas bentoníticas podem ser encontradas em várias ocorrências, sendo a mais estudada e conhecida a localizada no município de Boa Vista, PB. No entanto, a grande utilização e o elevado volume de extração de forma desordenada dessas argilas fizeram com que a maioria das variedades mineralógicas se exauriu. Por outro lado, recentemente, foram descobertos novos jazimentos de argilas esmectíticas no município de Cubati, que podem representar uma interessante alternativa tecnológica à futura escassez das argilas bentoníticas de Boa Vista.Entretanto, esses jazimentos ainda não foram estudados e caracterizados o que é necessário para a definição dos seus 
usos tecnológicos potenciais.

Dentre os principais usos das bentonitas destaca-se sua utilização em fluidos de perfuração. Fluídos de perfuração são definidos como fluidos de circulação usados em perfurações rotativas para desenvolver funções requeridas durante a operação de perfuração de poços de petróleo [7]. As bentoníticas sódicas ou policatiônicas, quando transformadas em sódicas pelo tratamento com carbonato de sódio, incham na presença de água, aumentando várias vezes seu volume inicial, sendo utilizadas no preparo de fluidos de perfuração devido às excelentes propriedades coloidais e tixotrópicas que apresentam [8]. As bentonitas melhoram as propriedades dos fluidos durante a operação de perfuração de poços, desempenhando uma ou várias das seguintes funções: aumentar a capacidade de limpeza do poço, reduzir as infiltrações nas formações permeáveis, formar uma membrana de baixa permeabilidade ("filtercake"), promover a estabilidade do poço e reduzir ou evitar perdas de circulação $[9,10]$. Os fluidos de perfuração são indispensáveis às atividades de perfuração de um poço, pois desempenham uma série de funções essenciais $[10,11]$, como: suspensão e remoção dos detritos gerados durante a perfuração, resfriar e limpar a broca, controle de pressão, estabilização das formações, lubrificação e resfriamento da broca, entre outras [10]. Assim, o estudo do comportamento reológico de dispersões de argilas bentoníticas é de grande interesse para indústria e pesquisadores.

Reologia é a ciência que estuda a deformação da matéria, no caso de corpos rígidos, ou o escoamento dos fluidos, no caso de sistemas líquidos ou gasosos. De modo bem abrangente os fluidos são classificados como newtonianos ou não newtonianos. São newtonianos os fluidos cuja viscosidade só é influenciada pela temperatura e pressão em regime laminar, ou seja, a razão entre a tensão de cisalhamento, $\tau$, e a taxa de cisalhamento, $\delta$,é uma constante, denominada viscosidade absoluta do fluido, $\mu[12,13]$. Em termos matemáticos:

$$
\tau / \delta=\mu
$$

O termo viscosidade pode ser entendido como "o atrito interno de um fluido" causado pela atração molecular, o qual provoca uma resistência ao escoamento. Todo fluido que não exibe uma proporcionalidade entre a tensão cisalhante e o gradiente de velocidade (taxa de cisalhamento), à temperatura e pressão constantes em regime laminar, é denominado não newtoniano. A viscosidade desse fluido, portanto, não é única e varia com a magnitude da tensão cisalhante aplicada $[12,13]$. Matematicamente tem-se:

$$
\tau / \delta=\mu_{\mathrm{a}}
$$

na qual a variável $\mu_{\mathrm{a}}$ é denominada viscosidade aparente.

Dispersões de sólidos em líquidos são em geral fluidos não newtonianos, principalmente quando os sólidos dispersos tendem a interagir (solvatar, inchar, etc.) com a fase líquida. Os fluidos de perfuração base água (suspensões de argila em água) são exemplos de fluidos não newtonianos [13]. $\mathrm{Na}$ literatura do escoamento de fluidos existe um variado número de equações matemáticas que podem ser utilizadas para descrever o comportamento de um fluido em particular [14]. Dentre esses modelos matemáticos, os mais aceitos para descrever o comportamento dos fluidos de perfuração base água utilizados na indústria do petróleo são: o modelo de Bingham ou plástico ideal, o modelo de Ostwald ou modelo de potência e o modelo de potência com limite de escoamento ou modelo de Hersclell-Bulkely [12, 13].

O modelo Binghamiano é definido pela equação:

$$
\tau=\mu_{\mathrm{p}}+\tau_{\mathrm{L}}
$$

na qual $\mu_{\mathrm{p}} \mathrm{e} \tau_{\mathrm{L}}$ são denominados viscosidade plástica e limite de escoamento, respectivamente. Fisicamente o limite de escoamento representa o valor mínimo de tensão cisalhante que deve ser aplicada para dar início ao escoamento do fluido Binghamiano.

O modelo de potência é definido pela equação:

$$
\tau=K(\delta)^{\mathrm{n}}
$$

na qual $K$ e n são parâmetros reológicos do modelo, denominados índice de consistência e índice de comportamento de fluxo, respectivamente. Quando o índice de comportamento $\mathrm{n}$ aproxima-se de 1 o fluido aproxima-se do modelo newtoniano e $\mathrm{K}$ coincide com a viscosidade. No entanto, quando os fluidos possuem valor de $\mathrm{n}$ menor que 1 são denominados pseudo-plásticos e sua viscosidade reduz com o aumento da taxa de cisalhamento. Quando os valores de $\mathrm{n}$ são superiores a 1 os fluidos são chamados de dilatantes e suas viscosidades aumentam com a taxa de cisalhamento $[13,14]$.

O modelo de potência com limite de escoamento é bem parecido com o modelo de potência com a única diferença sendo que esse modelo apresenta uma resistência ao início do escoamento, representada por $\tau_{0}$. A equação $E$ define esse modelo:

$$
\tau=K(\delta)^{\mathrm{n}}+\tau_{0}
$$

com $\mathrm{K}$, $\mathrm{n}$ e $\tau_{\mathrm{o}}$ constantes [13].

Deve-se mencionar que nenhum dos modelos citados levou em consideração o efeito do tempo na relação tensão de cisalhamento-taxa de cisalhamento. Contudo, existem fluidos que dependem do tempo, e são classificados como reopéticos e tixotrópicos. Nos reopéticos a tensão de cisalhamento aumenta com o tempo de fluxo, a uma mesma taxa de deformação, enquanto nos tixotrópicos há uma diminuição da tensão de cisalhamento com o tempo. Em geral os fluidos de perfuração base água não se ajustam perfeitamente a um dos modelos clássicos. Assim, é indicado abordar a modelagem do comportamento reológico do fluido com o modelo que mais se aproximar. Em geral, o comportamento dos fluidos segue mais de um modelo, conforme a faixa de rotação analisada e, em algumas faixas 
de rotacão, mais de um modelo é boa aproximação [12]. Isso faz com que durante a análise da reologia do sistema seja necessária a determinação de parâmetros de fluxo de mais de um modelo, que em geral são o Binghamiano e o de potência.

O presente trabalho tem por objetivo dá prosseguimento à caracterização de argilas bentoníticas de Cubati, PB, estudando o comportamento reológico das dispersões argilaágua de modo avaliar seu desempenho para fins de fluido de perfuração de poços de petróleo base água.

\section{MATERIAIS E MÉTODOS}

Foram estudadas argilas bentoníticas policatiônicas provenientes da Fazenda Campos Novos, município de Cubati, PB. As amostras foram identificadas por meio de numeração, apresentada na Tabela I e também a designação usada para identificar as argilas na área de extração.

Tabela I - Numeração utilizada e a designação usada no município de Cubati, PB.

[Table I - Adopted numeration and designation used in Cubati, PB.]

\begin{tabular}{cc}
\hline Numeração Utilizada & Denominação Local \\
\hline Amostra 1-A1 & Abaixo da Cinza 2 \\
Amostra 2-A2 & Aluísio \\
Amostra 3 - A3 & Poço 02 \\
Amostra 4-A4 & Cinza ou PO 2 Cinza \\
Amostra 5 - A5 & Abaixo da Cinza 1 ou Amarela \\
Amostra 6-A6 & PO 1 \\
Amostra 8-A8 & P2 CL \\
\hline
\end{tabular}

Foi utilizado carbonato de sódio $\left(\mathrm{Na}_{2} \mathrm{CO}_{3}\right)$ anidro PA Vetec para transformação das argilas individuais e das composições binárias de argilas bentoníticas em sódicas. As amostras coletadas foram secas a $60^{\circ} \mathrm{C}$, passadas em peneira ABNT $200(0,074 \mathrm{~mm})$ e, em seguida, foram determinadas suas capacidades de troca de cátions pela técnica de adsorção de azul de metileno [15]. As argilas secas foram tratadas com solução concentrada de carbonato de sódio em proporções variando de 50 a $150 \mathrm{meq} / 100 \mathrm{~g}$ de argila e curadas por período de 5 dias, segundo procedimento adotado $[1,3]$, para transformação das argilas em bentonitas sódicas. Com as argilas tratadas foram preparadas dispersões argila-água com concentração de 4,86\%, em massa, segundo normalização [16] e determinadas suas curvas de defloculação. Esse procedimento consistiu em adicionar 24,3 $\mathrm{g}$ de argila em $500 \mathrm{~mL}$ de água deionizada, agitar durante 20 min a 17.000 rpm (agitadores Hamilton Beach, 936) e deixar em repouso por $24 \mathrm{~h}$. Após esse tempo as amostras foram agitadas por 5 min a $17.000 \mathrm{rpm}$ [16] e foram determinadas as viscosidades aparente (VA) e plástica (VP) das argilas em função do teor de carbonato de sódio. As viscosidades foram determinadas em viscosímetro Fann, 35A. Com base nas curvas de defloculação, foram determinados teores ótimos de carbonato de sódio e, em seguida, foi feito o estudo reológico de dispersões argila-água produzidas com as argilas aditivadas com os teores ótimos de carbonatos.

O estudo reológico foi desenvolvido com viscosímetro, com raio externo e interno de $1,8415 \mathrm{~cm}$ e $1,7245 \mathrm{~cm}$, respectivamente, e molar de torção F-1. Foram efetuadas leituras a 3,6,100,200, 300 e $600 \mathrm{rpm}$, conforme a metodologia de preparação das dispersões e agitação descrita anteriormente [16]. Com base nos valores determinados, as viscosidades, plásticas e aparente, o limite de escoamento, o índice de comportamento de fluxo e índice de consistência foram calculados. Para determinação dos dois últimos foi utilizado processo interativo até a convergência das equações. Também foi determinado o volume de filtrado em filtro prensa, com aplicação de uma pressão de $\sim 7,0 \mathrm{kgf} / \mathrm{cm}^{2}$ (100 psi) durante $30 \mathrm{~min}$ [16].

A fim de avaliar o comportamento tixotrópico das suspensões analisadas, foram construídas as curvas deflexão do viscosímetro-rotação aplicada, pela medida dos valores de deflexão com o aumento da rotação seguido pela diminuição da rotação (curva de histerese), com as velocidades de 3,6, $100,200,300$ e 600 rpm [16] e o procedimento de preparação das dispersões e agitação já descrito anteriormente. Foram construídas curvas deflexão, $\theta$ versus rotação $\mathrm{N}$, ao invés de curvas tensão cisalhante $\tau$ versus gradiente de velocidade $\gamma$, por existir uma proporção direta entre as grandezas $\theta$ e $\tau$ e entre $\mathrm{N}$ e $\gamma$, quando se trabalha com viscosímetro rotativo. Assim, por simplicidade preferiu-se $\theta$ versus $\mathrm{N}$ a fim de avaliar o fenômeno da tixotropia.

Para obtenção da força gel inicial, o fluido foi agitado a $600 \mathrm{rpm}$ durante $15 \mathrm{~s}$, a velocidade foi mudada para $3 \mathrm{rpm}$ e o fluido foi deixado em repouso durante $10 \mathrm{~s}$, e foi feita a leitura obtendo o valor da força gel inicial. Em seguida, para a obtenção da força gel final, o fluido foi deixado em repouso durante 10 min e efetuada a leitura a $3 \mathrm{rpm}$.

\section{RESULTADOS E DISCUSSÃO}

Na Tabela II são apresentadas as capacidades de troca de cátions (CTC) das argilas analisadas, segundo a metodologia de adsorção de azul de metileno. Os valores obtidos são inferiores aos valores de CTC observados em bentonitas sulamericanas [17, 18]. Estes valores podem estar associados, em parte, ao elevado teor de minerais acessórios e impurezas nas bentonitas estudadas, tal como já evidenciado [19].

Tabela II - Capacidade de troca de cátions determinada pelo método de azul de metileno.

[Table II - Cation exchange capacity (CEC) determined by methylene blue adsorption methodology.]

\begin{tabular}{lccccccc}
\hline Amostra & A1 & A2 & A3 & A4 & A5 & A6 & A8 \\
\hline $\begin{array}{l}\text { CTC } \\
\text { (meq/ }\end{array}$ & 37,01 & 23,13 & 32,38 & 43,95 & 32,38 & 27,76 & 25,45 \\
100g) & & & & & & & \\
\hline
\end{tabular}




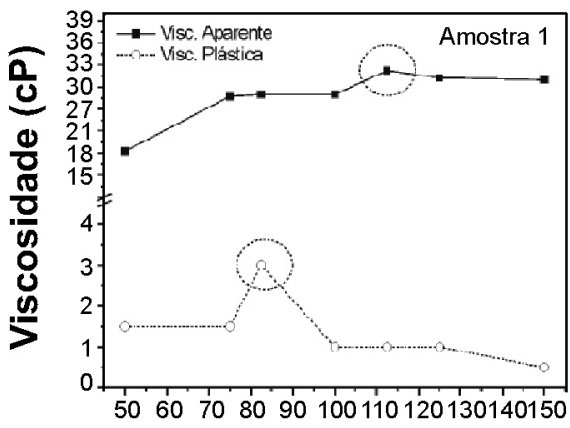

Teor de $\mathrm{Na}_{2} \mathrm{CO}_{3}$ (meq/100g)

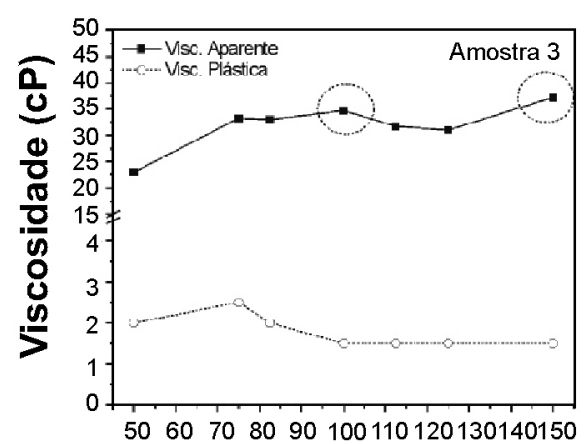

Teor de $\mathrm{Na}_{2} \mathrm{CO}_{3}$ (meq/100g)

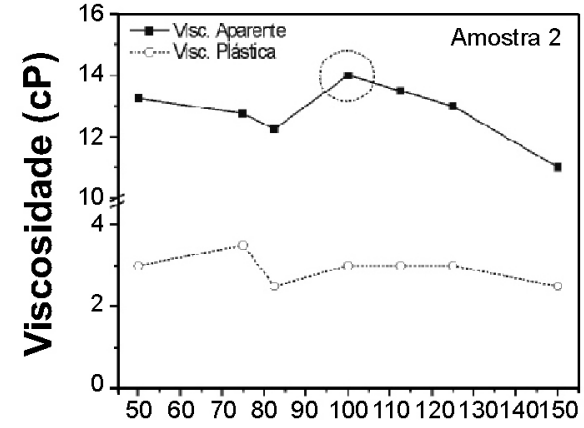

Teor de $\mathrm{Na}_{2} \mathrm{CO}_{3}$ (meq/100g)

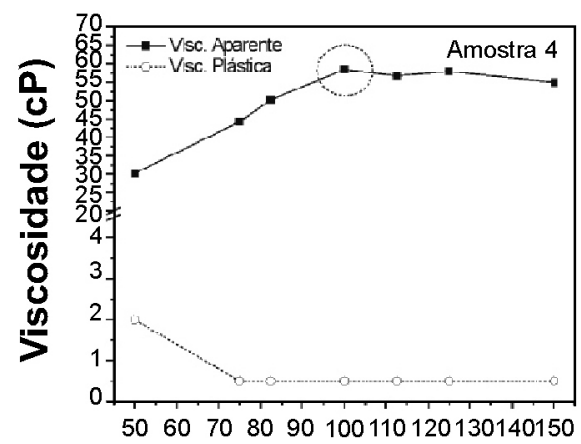

Teor de $\mathrm{Na}_{2} \mathrm{CO}_{3}$ (meq/100g)

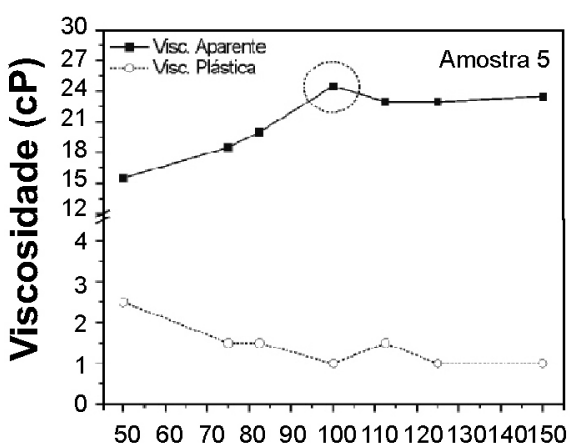

Teor de $\mathrm{Na}_{2} \mathrm{CO}_{3}$ (meq/100g)

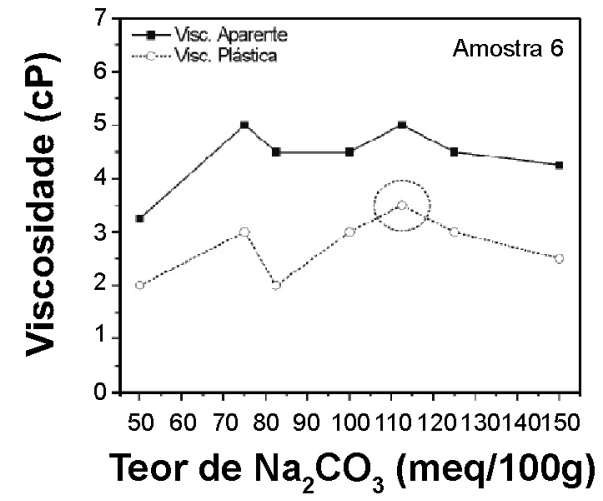

Teor de $\mathrm{Na}_{2} \mathrm{CO}_{3}$ (meq/100g)

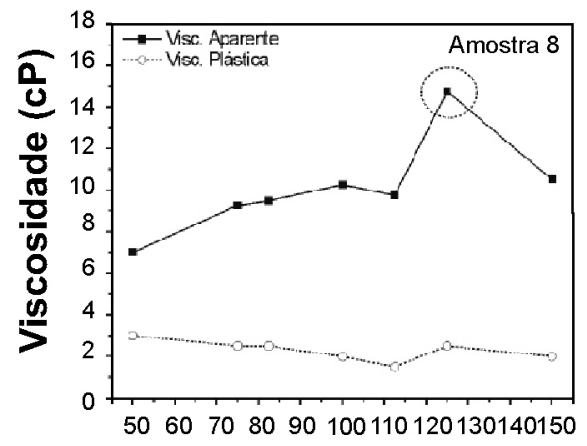

Teor de $\mathrm{Na}_{2} \mathrm{CO}_{3}(\mathrm{meq} / 100 \mathrm{~g})$

Figura 1: Curvas de defloculação das amostras estudadas. [Figure 1: Deflocculation curves of the studied samples.] 
Na Fig. 1 são apresentadas as curvas de dispersão das amostras analisadas em função do teor de carbonato de sódio utilizado durante o processo de transformação em sódicas. As viscosidades, aparente (VA) e plástica (VP), variaram significativamente em função do teor de carbonato utilizado. Não se observa um comportamento bem definido da viscosidade aparente com relação ao teor de carbonato, de forma geral, a viscosidade aumentou até um máximo (ou faixa de valores) e decresceu em seguida. No que se refere à viscosidade plástica não se constatou nenhuma tendência de comportamento. Foram escolhidos teores de carbonato de sódio para cada argila de acordo com os maiores valores de viscosidade aparente e plástica obtidos. Essas seleções estão indicadas na Fig. 1 por meio de círculos e são apresentadas na Tabela III, juntamente com a denominação utilizada para identificá-las.

Tabela III - Designação das amostras após tratamento com $\mathrm{Na}_{2} \mathrm{CO}_{3}$.]

[Table III - Samples designation after activation with $\mathrm{Na}_{2} \mathrm{CO}_{3}$.]

\begin{tabular}{cc}
\hline Denominação & $\begin{array}{c}\text { Amostra - Teor de } \mathrm{Na}_{2} \mathrm{CO}_{3} \\
\text { utilizado no tratamento }\end{array}$ \\
\hline A1 82,5 & $1-82,5$ meq de $\mathrm{Na}_{2} \mathrm{CO}_{3} / 100 \mathrm{~g}$ \\
A1 112,5 & $1-112,5$ meq de $\mathrm{Na}_{2} \mathrm{CO}_{3} / 100 \mathrm{~g}$ \\
A2 100 & $2-100$ meq de $\mathrm{Na}_{2} \mathrm{CO}_{3} / 100 \mathrm{~g}$ \\
A3 100 & $3-100$ meq de $\mathrm{Na}_{2} \mathrm{CO}_{3} / 100 \mathrm{~g}$ \\
A3 150 & $3-150$ meq de $\mathrm{Na}_{2} \mathrm{CO}_{3} / 100 \mathrm{~g}$ \\
A4 100 & $4-100$ meq de $\mathrm{Na}_{2} \mathrm{CO}_{3} / 100 \mathrm{~g}$ \\
A5 100 & $5-100$ meq de $\mathrm{Na}_{2} \mathrm{CO}_{3} / 100 \mathrm{~g}$ \\
A6 112,5 & $6-112,5$ meq de $\mathrm{Na}_{2} \mathrm{CO}_{3} / 100 \mathrm{~g}$ \\
A8 125 & $8-125$ meq de $\mathrm{Na}_{2} \mathrm{CO}_{3} / 100 \mathrm{~g}$ \\
\hline
\end{tabular}

Foram preparadas dispersões argila-água utilizando as amostras indicadas na Tabela II (com as argilas sendo aditivas com seus os teores ótimos de carbonato de sódio). Esses fluidos foram caracterizados através da determinação de suas propriedades reológicas em viscosímetro rotativo de cilindros concêntricos. Como mencionado anteriormente, os fluidos de perfuração podem ser estudados através da escolha dos modelos matemáticos mais adequados, sendo os mais aceitos, para descrever seu comportamento no estado sol os de Bingham e Ostwald. Os dois modelos constituem boas aproximações, todavia nenhum cobre todo o reograma apresentado por esses fluidos. Assim, é necessária a determinação de propriedades reológicas utilizando tanto o modelo de Bingham quanto o de Ostwald para a adequada compreensão do comportamento dos fluidos. Com base no modelo Binghamiano foram determinados a viscosidade plástica e o limite de escoamento dos fluidos analisados no intervalo convencional da API (300 a 600 rpm) [16]. Na Tabela IV são apresentadas essas propriedades reológicas. As dispersões apresentaram valores de viscosidade plástica, semelhantes aos das argilas bentoníticas de Boa Vista, PB
[17], utilizadas há vários anos como agentes viscosificantes em fluidos de perfuração base água. Valores superiores a $4 \mathrm{cP}$ para dispersões de argilas bentonitas para fins de aplicação em fluidos de perfuração base água são especificados [20]. No entanto verifica-se que esses valores podem ser facilmente corrigidos, atingindo os valores limites da normalização, com pequenas adições poliméricas [5, 8]. As dispersões apresentaram limites de escoamento variando de 1,43 a 55,54 Pa, não sendo observada correlação entre o limite e a viscosidade plástica, o que pode se dever ao fato do limite de escoamento relacionar-se às forças de interação de natureza elétrica partícula-partícula, enquanto a viscosidade plástica ser o resultado do atrito partícula-partícula e fluido-fluido [13].

Também foram calculados os valores de viscosidade aparente e limite de filtrado das dispersões. Os resultados são apresentados na Tabela IV. A viscosidade aparente variou de 5 a $58 \mathrm{cP}$, enquanto que o volume de filtrado de 16 a $32 \mathrm{~mL}$. O limite inferior de $15 \mathrm{cP}$ para a viscosidade aparente e de superior de $18 \mathrm{~mL}$ para o volume de filtrado é definido [20]. Assim, apenas as amostras A3 e A4 apresentaram valores dessas propriedades de acordo com as especificações. Espera-se que quanto mais floculado esteja o sistema analisado, maior sua viscosidade aparente e também menor o volume de filtrado, em virtude de uma maior retenção de água pelo sistema. Não há uma relação bem definida entre a viscosidade aparente e o volume de filtrado; no entanto há uma tendência clara de aumento do volume com a diminuição da viscosidade. Considerando que as dispersões seguem o modelo de Ostwald no intervalo de 300 a $600 \mathrm{rpm}$, foram determinados os valores do índice de comportamento de fluxo, n, e de consistência, K. Seus valores são apresentados na Tabela IV. Os valores do índice de comportamento variaram de 0,03 a 0,62 , no entanto, todos se situaram abaixo de 1 , indicando que se tratam de

Tabela IV - Propriedades reológicas e de filtração de suspensões argila-água preparadas com as bentonitas.

[Table IV - Rheological and filtration properties of waterclay suspensions prepared with the bentonites.]

\begin{tabular}{ccccccc}
\hline Amostras & $\begin{array}{c}\mathrm{VP} \\
(\mathrm{cP})^{\mathrm{a}}\end{array}$ & $\begin{array}{c}\tau_{\mathrm{L}} \\
(\mathrm{Pa})^{\mathrm{b}}\end{array}$ & $\begin{array}{c}\mathrm{VA} \\
(\mathrm{cP})^{\mathrm{c}}\end{array}$ & $\begin{array}{c}\mathrm{VF} \\
(\mathrm{mL})^{\mathrm{d}}\end{array}$ & $\mathrm{n}^{\mathrm{e}}$ & $\begin{array}{c}\mathrm{K} \\
\left(\mathrm{Pa} \mathrm{s}^{\mathrm{n}}\right)^{\mathrm{f}}\end{array}$ \\
\hline 182,5 & 3,0 & 24,90 & 29,0 & 22,2 & 0,077 & 7,91 \\
1112,5 & 1,0 & 29,92 & 32,2 & 21,9 & 0,023 & 12,96 \\
2100 & 3,0 & 10,53 & 14,0 & 27,0 & 0,163 & 2,09 \\
3100 & 1,5 & 31,84 & 34,7 & 16,0 & 0,031 & 13,06 \\
3150 & 1,5 & 34,23 & 37,2 & 18,8 & 0,029 & 14,22 \\
4100 & 0,5 & 55,54 & 58,5 & 18,0 & 0,006 & 26,87 \\
5100 & 1,0 & 22,50 & 24,5 & 21,8 & 0,030 & 9,33 \\
6112,5 & 3,5 & 1,43 & 5,0 & 32,5 & 0,621 & 0,03 \\
8125 & 2,5 & 11,73 & 14,7 & 30,5 & 0,128 & 2,82 \\
\hline
\end{tabular}

${ }^{a}$ Viscosidade plástica, ${ }^{b}$ Limite de escoamento, 'Viscosidade aparente, 'Volume de Filtrado, éndice de comportamento de fluxo, fíndice de consistência 
dispersões pseudo-plásticas e não dilatantes. Os valores obtidos para o índice de consistência são semelhantes a valores determinados para fluidos de perfuração de poços de petróleo base água.

Os fluidos à base de água e argila possuem comportamento reológico dependente não apenas da taxa de cisalhamento (pseudo-plásticos e dilatantes), mas também do tempo, conhecido como tixotropia. Os fluidos tixotrópicos apresentam um incremento de viscosidade quando em condições estáticas (em repouso) pela formação do estado gel, e recuperação da fluidez (estado sol), quando submetido à condições dinâmicas (cisalhamento) [1]. A maioria dos fluidos de perfuração à base água exibe essa propriedade devido ao surgimento de forças atrativas entre as partículas de argilas, à baixa taxa de cisalhamento (fluxo lento) ou em repouso. Essa atração é possível porque a distribuição de cargas elétricas sobre a superfície das partículas de argilas não é uniforme, apresentando cargas residuais negativas nas faces e positivas nas arestas [13].

A Fig. 2 apresenta as curvas de deflexão versus rotação das dispersões analisadas. Não foi analisado o comportamento tixotrópico da dispersão contendo a amostra A6 por apresentar valor de viscosidade aparente muito baixo e volume de filtrado muito elevado, o que não indica para utilização em fluidos de perfuração base água. Podese observar um comportamento de histerese das curvas, com a curva de deflexão não retornando a posição inicial com a diminuição da rotação. O que, possivelmente está relacionado ao fenômeno de tixotropia em baixas taxas de cisalhamento (3 e 6 rpm), ocorrendo interação face-to-edge

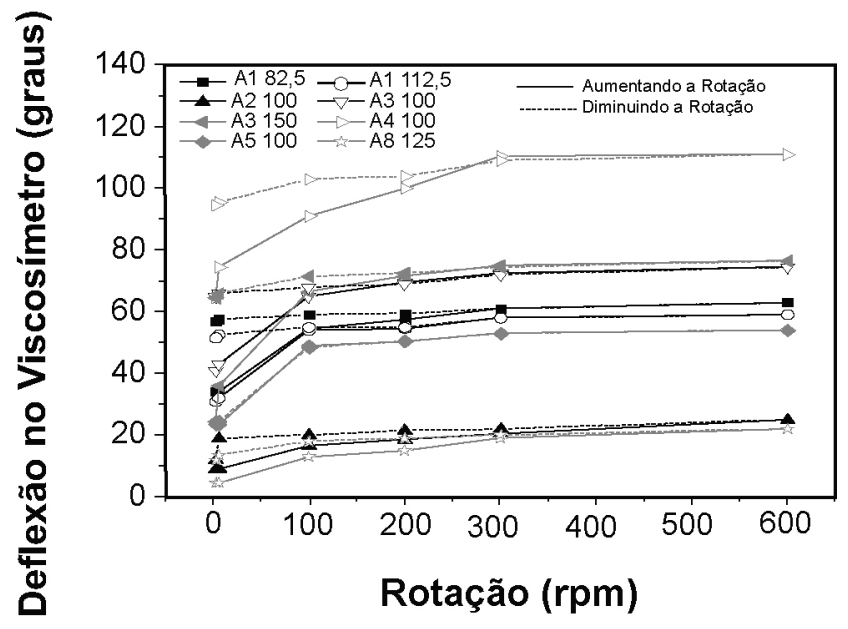

Figura 2: Curvas de fluxo das dispersões analisadas.

[Figure 2: Flowcharts of the analyzed dispersions.] entre as partículas e por conseguinte aumento da viscosidade, retratado na maior deflexão, com o transcorrer do tempo necessário para a estabilização do defletor do viscosímetro e a efetuação da leitura.

As amostras A1, A3 e A4 foram as que apresentaram as maiores histereses, indicativo de maior desenvolvimento de tixotropia. São também essas amostras as que apresentaram os maiores valores para o limite de escoamento. $\mathrm{O}$ que vem ao encontro do comentado anteriormente, já que o limite de escoamento é influenciado majoritariamente pela interação resultante de forças de natureza elétrica. Assim, as suspensões de todas as amostras, à exceção da $\mathrm{A} 8$, exibem fenômeno de tixotropia e não de reopexia. No entanto, outra maneira de analisar e buscar quantificar a magnitude desse fenômeno é através da força gel, que representa a força resistiva ao escoamento após certo tempo de repouso, sendo dada pela diferença entre as tensões de cisalhamento em uma taxa de cisalhamento zero (o que seria efetivamente limites de escoamento) em um tempo $i$ e em um tempo $f$, algum tempo após a primeira medida (tempo durante o qual a suspensão permaneceu em repouso). A Tabela V apresenta os valores calculados para a força gel, conforme a equação (F) [13], utilizando-se ao invés da medida na rotação zero (taxa de cisalhamento zero) o valor na rotação de 3 rpm:

$$
\tau_{\mathrm{i}}=0,51 \theta_{\mathrm{i}}
$$

na qual $\tau_{\mathrm{i}}$ é a tensão de cisalhante no tempo i e $\theta$ a deflexão medida no viscosímetro na rotação de $3 \mathrm{rpm}$ no tempo i.

As amostras A1, A3 e A4 são as que apresentam maior potencial tixotrópico. No entanto, é interessante observar que há uma grande mudança no comportamento da amostra A3 conforme o aumento no teor de carbonato de sódio usado para a troca catiônica. A amostra A3 150 apresenta uma força gel cerca de sete vezes superior a da amostra A3 100, apesar de possuir valores semelhantes de viscosidade aparente e limite de escoamento. Essas amostras apresentaram valores de viscosidade e limite de escoamento que indicaram que possivelmente apresentariam características de tixotropia distintas, mas muito parecidas, o que não ocorreu, evidenciando-se assim a necessidade de uma total caracterização reológica das dispersões para um real entendimento do seu comportamento. O comportamento distinto das amostras pode estar associado a uma grande concentração de íons sódio na superfície de argila, que apesar de não influenciar tanto no limite de escoamento, pode estar influenciando em muito no desenvolvimento da estrutura de castelo de cartas [1], formando uma estrutura floculada gel muito rapidamente. A caracterização reológica

Tabela V - Força gel ${ }^{a}$ das dispersões analisadas. [Table V-Gel force of the analyzed dispersions.]

\begin{tabular}{ccccccccc}
\hline Amostra & A1 82,5 & A1 112,5 & A2 100 & A3 100 & A3 150 & A4 100 & A5 100 & A8 125 \\
\hline Força Gel $\left(\mathrm{N} / \mathrm{mm}^{2}\right)^{\mathrm{a}}$ & 1,0 & 0,5 & 0,3 & 0,3 & 2,3 & 0,8 & 0,5 & 0,0 \\
\hline${ }^{a}$ Resistência do estado gel ao escoamento, determinada em & rpm. & & & & & &
\end{tabular}


se mostra extremamente importante para a descoberta do comportamento viscosificante das bentonitas de Cubati, $\mathrm{PB}$, em fluidos de perfuração de poços de petróleo base água, o que, aliado à sua caracterização físico-mineralógica apresentada anteriormente [19], evidencia que essas bentonitas apresentam grande potencial para aplicação na tecnologia do petróleo, possuindo características reológicas similares as das bentonitas de Boa Vista, PB, que são muito utilizadas para esse fim.

\section{CONCLUSÃO}

As argilas estudadas apresentam pequena capacidade de troca de cátions, segundo a metodologia de adsorção de azul de metileno; o teor ótimo de carbonato de sódio usado para a troca catiônica das bentonitas varia de acordo com a amostra, de 82,5 a $150 \mathrm{meq} / 100 \mathrm{~g}$ de argilas; as dispersões argila-água apresentam comportamento pseudo-plástico e tixotrópico; e duas amostras possuem grande potencial para utilização como agentes viscosificantes para fluidos de perfuração.

\section{AGRADECIMENTOS}

Ao CNPq (Proc. 550653/2007-4) e à FAPESQ/MCT/ CNPq (Projeto DCR 001/06), pelo apoio financeiro.

\section{REFERÊNCIAS}

[1] P. Souza Santos, Ciência e Tecnologia de Argilas, vol. 1, $2^{a}$ Ed., Edgar Blücher, S. Paulo, SP (1992).

[2] G. Lagaly, Clay Miner. 16 (1981) 1.

[3] A. R. Zandonadi, Estudo tecnológico de argilas montmoriloníticas brasileiras, Tese de Doutorado, Instituto de Química da USP, S. Paulo, SP (1972).

[4] R. F. Valenzuela Díaz, P. Souza Santos, H. Souza Santos, Quím. Ind. 42 (1992) 33.
[5] L. V. Amorim, K. V. Farias, J. D. Viana, M. I. R. Barbosa, E. Pereira, K. B. França, H. L. Lira, H. C. Ferreira, Cerâmica 51 (2005) 128.

[6] H. H. Murray, Appl. Clay Sci. 17 (2000) 207.

[7] J. L. Lummus, J. J. Azar, Drilling fluids optimization: a practical field approach, PennWell Publ. Co., Tulsa, EUA (1986).

[8] M. I. R. Barbosa, L. V. Amorim, H. C. Ferreira, Cerâmica $\mathbf{5 3}$ (2007) 354.

[9] H. C. H. Darley, G. R. Gray, Composition and properties of drilling and completion fluids, $5^{\text {th }}$ Ed., Gulf Publ. Co., Houston, EUA (1988).

[10] L. V. Amorim, C. M. Gomes, F. L. H. Silva, H. C. Ferreira, Cerâmica 48 (2002) 234.

[11] R. Caenn, G. V. Chillingar, J. Petroleum Sci. Eng. 14 (1996) 221.

[12] S. Amui, Reologia, Salvador, Petrobrás, Divisão de Ensino (1979).

[13] J. C. Machado, Reologia de Fluidos: Aplicação na Engenharia de Petróleo, Salvador, Petrobrás (1983).

[14] R. F. Navarro, Fundamentos de Reologia de Polímeros, EDUCS, Caxias do Sul, RS (1997).

[15] H. C. Ferreira, T. Chen, A. R. Zandonadi, P. Souza Santos, Cerâmica 18 (1972) 333.

[16] Petrobrás, Ensaio de viscosificante para fluido de perfuração base de água na exploração e produção de petróleo, Método, N-2605, Rio de Janeiro, RJ (1998).

[17] L. V. Amorim, C. M. Gomes, H. L. Lira, K. B. França, H. C. Ferreira, Mater. Res. 7, 4 (2004) 583.

[18] A. M. M. Carrera, A. F. D. C. Varajão, M. A. Gonçalves, REM: Rev. Escola de Minas 61, 1 (2008) 97.

[19] R. R. Menezes, P. M. Souto, L. N. L. Santana, G. A. Neves, R. H. G. A. Kiminami, H. C. Ferreira, Cerâmica 55, 334 (2009) 163.

[20] Petrobrás, Viscosificante para fluido de perfuração base de água na exploração e produção de petróleo, Especificação $\mathrm{N}-2604$. Rio de Janeiro, RJ (1998).

(Rec. 10/10/2008, Ac. 13/12/2009) 